La

Révolution

française

\section{La Révolution française}

Cahiers de l'Institut d'histoire de la Révolution française

5 | 2013

Le républicanisme anglais dans la France des

Lumières et de la Révolution

\title{
Essai sur la réception de l'œuvre de Thomas Gordon dans la France des Lumières
}

Eric Gasparini

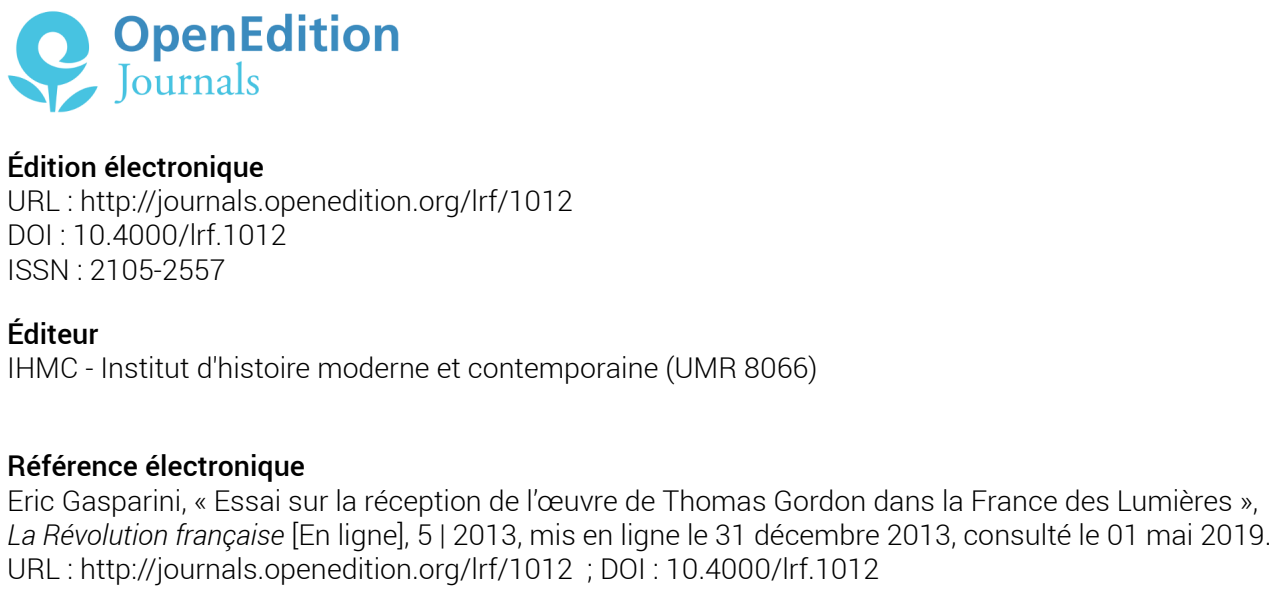

Ce document a été généré automatiquement le 1 mai 2019.

(c) La Révolution française 


\title{
Essai sur la réception de l'œuvre de Thomas Gordon dans la France des Lumières
}

\author{
Eric Gasparini
}

1 Thomas Gordon est connu aujourd'hui pour ses Cato's Letters, rédigées entre 1720 et 1723 en collaboration avec John Trenchard et qui allaient constituer une source fondamentale de la Commonwealthman tradition ${ }^{1}$ puis des révolutionnaires américains d'Adams à Jefferson. Souvent minorée, l'influence de ses œuvres en France au XVIII ${ }^{\mathrm{e}}$ siècle mérite pourtant de faire l'objet d'une lecture renouvelée.

2 Né trois ans environ après la Glorious Revolution de 1688, Gordon appartient à ce qu'on peut considérer comme la troisième génération du républicanisme anglais, celle des néoharringtoniens ${ }^{2}$ - la référence à Harrington s'avérant centrale ${ }^{3}$ - parmi lesquels John Toland, Robert Molesworth, ou encore Walter Moyle. Ces derniers, considérés comme des «Country Whigs » travaillent la dimension éthique du républicanisme en s'intéressant aux notions de liberté, de vertu, de commerce et de corruption ${ }^{4}$.

3 La vie de Thomas Gordon est mal connue. Un Thomas Gordon aurait été diplômé du King's College en 1713, et on a également retrouvé un Thomas Gordon soumettant une thèse en droit devant l'université d'Edimbourg en $1716^{5}$. Mais s'agit-il bien de notre auteur? Gordon se fait connaître comme collaborateur de John Trenchard en tant que rédacteur principal de l'hebdomadaire The Independant Whig dont les articles vont être rassemblés en un volume en 1721 et connaître plusieurs éditions successives ${ }^{6}$. L'ouvrage, animé par un fort anticléricalisme, est une attaque en règle du High Church Party et des prétentions exorbitantes du clergé proche des conservateurs. Cet ouvrage sera d'ailleurs traduit en français par le Baron d'Holbach'.

Dans le même temps, Gordon commence avec Trenchard que l'on peut considérer comme son mentor la rédaction de la série des Cent quarante-quatre Lettres sur Caton qui s'achèvent avec la mort de John Trenchard en $1723^{\circ}$. Ce dernier, membre du Parlement depuis les années 1690 s'était notamment illustré avec Walter Moyle lors de la controverse autour de la standing $a r m y^{9}$. Le rejet d'une armée permanente et soldée étant 
l'une des antiennes du républicanisme anglais. Certains historiens estiment que Trenchard et Gordon appartiennent à cette époque au courant des Real Whigs, qui considèrent que les libertés anglaises et le libre gouvernement sont menacés par certaines pratiques dont notamment la corruption ${ }^{10}$.

Les Lettres sur Caton condamnent la corruption du gouvernement Walpole et le manque de moralité qui entache le système politique britannique. L'œuvre développe des idées assez proches à cet égard de l'opposant conservateur Bolingbroke, réunies dans le Patriot King ${ }^{11}$. De plus, Trenchard et Gordon y enrichissent le thème de la vertu civique par l'évocation des droits de l'homme et du contrat social ${ }^{12}$.

L'opposition affichée de Thomas Gordon à Walpole cesse toutefois en 1723. Le Premier Ministre s'en attache les services en lui offrant la place de premier commissaire des licences de vin, poste qu'il occupera jusqu'à sa mort en 1750.

7 C'est durant cette période que Gordon approfondit sa connaissance de l'Histoire romaine en traduisant en langue anglaise les œuvres de Tacite auxquelles il ajoute des commentaires critiques sur le modèle de ceux qu'avait rédigés Machiavel sur Tite-Live. L'ouvrage est publié en 1728 sous le titre de The works of Tacitus With Political Discourses, et il est dédié à Walpole ${ }^{13}$. Très influencé par les idées républicaines d'Harrington et de Sidney, Gordon ne revendique pas pour autant l'épithète de "républicain », le mot étant obscurci par le souvenir de la dictature de Cromwell. Gordon publiera également dans la même veine des discours politiques sur Salluste en $1744^{14}$. C'est surtout par l'intermédiaire de ces deux œuvres que Gordon a été connu et apprécié des lecteurs français.

8 Tout au long du XVIII ${ }^{e}$ siècle, et durant la période qui précède la Révolution française, la connaissance de l'auteur écossais est avérée en France. Ses œuvres sont connues, disséquées, par un milieu lettré, avide des réflexions portées par «ce vent nouveau qui souffle d'Angleterre » selon les propres mots du marquis d'Argenson en 1751. Sans doute la traduction française de ses ouvrages sur Tacite et Salluste élaborée par le protestant Pierre Daudé joue-t-elle pour beaucoup dans leur diffusion chez les Français ${ }^{15}$. Mais l'anglophilie de certains grands noms de la République des Lettres françaises, comme Voltaire ou Montesquieu, peut également expliquer la diffusion des idées de Gordon.

9 La lecture républicaine faite par Gordon de l'œuvre de Tacite a pu ainsi séduire les opposants français à l'absolutisme monarchique. Et s'ils ne constituent pas un «programme républicain » à proprement parler, les Discours, selon les propres mots de l'auteur dans l'introduction de son ouvrage, ont bien été composés pour « défendre la liberté publique contre les violences du gouvernement et les injustices de ceux qui ont l'autorité16..$"$ Ce n'est donc pas un hasard si un arrêt du Parlement de Toulouse du 26 août 1751 ordonne que le "livre intitulé Discours historiques, critiques et politiques sur Tacite sera lacéré et brûlé par la main de l'exécuteur de la Haute-Justice ${ }^{17}$. » Mais l'œuvre de Thomas Gordon s'inscrit également au XVIII siècle dans un mouvement intellectuel plus large qui remet l'histoire romaine à l'honneur, thème dont étaient particulièrement férus les lettrés français de l'époque ${ }^{18}$. La France des Lumières est alors en pleine querelle entre germanistes et romanistes. Cette "prégnance de Rome ", qui confine à l'obsession chez les auteurs français ${ }^{19}$, s'accompagne d'une véritable mode en faveur de Tacite et de Salluste, dont les préceptes moraux pouvaient nourrir une véritable réflexion politique ${ }^{20}$. 
10 Les recensions des Discours sur Tacite faites à dix années d'intervalle par des auteurs français dans deux gazettes hollandaises témoignent en tous les cas de l'intérêt du public lettré de leur pays.

11 En 1733, c'est tout d'abord la Bibliothèque Britannique ou Histoire des ouvrages des savants de la Grande-Bretagne qui fait un très long compte rendu des deux volumes de l'ouvrage dans son édition en langue anglaise ${ }^{21}$ à Londres chez l'éditeur Thomas Woodward. L'auteur témoigne de l'importance acquise par Gordon en Angleterre. Ses premières œuvres lui ont assuré outre-Manche " beaucoup de réputation ${ }^{22}$. » Dans les Works of Tacitus, Gordon a joint «l'expérience dans les affaires de l'Etat à l'étude de l'Histoire \& de la morale ${ }^{23}$.» Selon l'auteur du compte rendu, il fait un portrait de Tacite parmi les plus avantageux. La méthode utilisée par l'auteur anglais est vantée : non seulement « les Annales et l'Histoire de Tacite ont fourni un beau champ [...] Mr. Gordon ne s'en est pas contenté : il a recueilli un bon nombre d'observations dans d'autres historiens anciens \& modernes, pour enrichir \& orner ces discours ${ }^{24}$.» De même, il pratique une approche comparatiste, mettant en symétrie les règnes de Tibère et Caligula et celui de Jacques II Stuart. Les idées politiques dont Gordon fait la promotion sont mises en exergue : on y retrouve pêle-mêle les antiennes du républicanisme Anglais: l'horreur de la tyrannie, le consentement nécessaire des peuples, le droit de résistance ${ }^{25}$, la limitation du gouvernement par les lois, la condamnation de la corruption des ministres, l'apologie de la vertu, le rejet des armées mercenaires et permanentes, et la condamnation de l'esprit de conquête ${ }^{26}$.

12 En 1742, Louis de Jaucourt et Armand Boisbeleau de la Chapelle livre dans la Bibliothèque raisonnée des ouvrages des savants de l'Europe une nouvelle analyse des Discours sur Tacite ${ }^{27}$. Mais cette fois, c'est la traduction faite par Pierre Daudé qui est examinée. L'engagement du Refuge Huguenot dans la diffusion en France des idées anglaises n'est plus à démontrer, les réfugiés protestants ayant largement diffusé la philosophie politique et les institutions anglaises dans l'opinion publique française ${ }^{28}$. Pierre Daudé est ainsi largement représentatif de cet esprit lorsqu'il traduit les Discours sur Tacite en 1742 et ceux sur Salluste en $1759^{29}$. L'exorde qui précède l'analyse de de Jaucourt et de Boisbeleau de la Chapelle est enthousiaste. En effet, l'ouvrage de Gordon a, selon eux, une ambition : "réprimer la tyrannie des Grands, \& empêcher que le plus faible ne soit opprimé par le plus fort [...] L'Auteur (Thomas Gordon) est un homme élevé au milieu d'une Nation extrêmement jalouse de ses Privilèges \& de ses Droits, qui semble ne respirer que la liberté, \& qui crie hautement à l'oppression lorsqu'elle se voit lésée dans la moindre de ses Prérogatives . C'est chez cette Nation que ceux qui aiment la liberté, \& par conséquent la félicité du Genre Humain, doivent aller chercher leurs Défenseurs ${ }^{30}$.» Jaucourt et son co-auteur mettent en relief la manière dont Gordon traite de la religion ce qui sera d'ailleurs revisité par d'autres commentateurs français. Selon eux, Gordon défend l'idée "qu'un Etat peut être gouverné par des hommes qui ne sont d'aucune religion, d'aucune secte, guidés uniquement par les lumières de la Raison \& d'une bonne morale $^{31}$.» Sur ce sujet, ils opèrent un rapprochement entre Gordon et Pierre Bayle ${ }^{32}$, notamment sur la question du gouvernement de l'Eglise : «Si ce grand philosophe vivait aujourd'hui, il serait ravi de voir son sentiment défendu par un aussi habile homme que Mr. Gordon ${ }^{33}$.» Ruse de l'histoire quand on sait que Pierre Bayle a reproché aux auteurs du Refuge de vulgariser les thèses républicaines anglaises et que Gordon s'est montré extrêmement critique à son encontre. Bien entendu, le commentaire de 1742 insiste sur les principes politiques qui irriguent la pensée de l'auteur écossais. Lorsque ce dernier traite du « Gouvernement libre \& du Gouvernement arbitraire », il établit « d'excellents 
principes $^{34}$.» Toutefois, il n'est pas exempt de critiques. En effet, les principes de Gordon pourraient être mis en pratique «si les hommes étaient tels qu'ils devraient être. » Mais aux yeux de Jaucourt et Boisbeleau, Gordon semble ne pas assez tenir compte des réalités sociales du temps. Ainsi, "vouloir réformer aujourd'hui sur son plan la plupart des gouvernements monarchiques n'aboutirait qu'à y jeter le désordre et la confusion ${ }^{35}$. » Le républicanisme affiché par l'auteur écossais pourrait mener à l'anarchie «\& donner lieu aux plus grands maux. » Jaucourt et son commensal poursuivent : «J'ose même dire que cette Doctrine prêchée et poussée un peu loin dans une République serait très dangereuse \& pourrait faire naître les plus affreux désordres ${ }^{36}$.» Pour les commentateurs français, " est-il utile de résister au Magistrat sous prétexte de quelques abus. Le principe de Mr. Gordon n'est bon que lorsqu'un Prince ou le Magistrat anéantit toutes les Loix, \& foule aux pieds toutes les règles de la Justice ${ }^{37}$. » Suit d'ailleurs un éloge de Louis XIV, éreinté par Gordon dans ses Discours, Louis XIV à qui «la France est redevable de sa grande puissance \& de cette force que l'on doit regarder comme l'effet d'un bon \& sage gouvernement ${ }^{38}$.» Il est évident que Jaucourt et Boisbeleau ne peuvent ouvertement suivre Gordon quand celui compare l'Angleterre, dont le gouvernement est libre, à la France qu'il classe dans les Etats « arbitraires » niant la liberté ${ }^{39}$. A l'inverse, Jaucourt et consort reprennent la critique du penseur écossais de l'esprit de conquête citant l'exemple de la rivalité russo-suédoise entre Charles XII et Pierre le Grand ${ }^{40}$.

Entre ces deux commentaires, Voltaire a publié en 1734 ses Lettres Philosophiques ou Lettres Anglaises. Dans ce livre, le philosophe français s'interroge sur la qualité des historiens britanniques : «Il est vrai qu'il y a à présent un Mr. Gordon, excellent traducteur de Tacite, très capable d'écrire l'histoire de son pays [...] mais Mr. Rapin (il s'agit de RapinThoiras) l'a prévenu ${ }^{41}$. » Dans une lettre du $1^{\mathrm{er}}$ septembre 1738 adressée à son protecteur d'alors, le futur Frédéric II de Prusse, Voltaire conseille au Prince qui rédige son AntiMachiavel de lire les discours de Gordon et sa traduction de Tacite, «bien dignes d'être vus $^{42}$.» Au passage, il est plaisant de voir Voltaire vanter à Frédéric II un auteur qui s'inscrit résolument dans le « moment machiavélien »! Voltaire fera plus tard référence à Gordon dans son Histoire de l'établissement du christianisme, en 1764. Il l'associe alors à la liste des "Free-thinkers, les francs pensants ${ }^{43}$ " aux côtés de Toland, Trenchard, Bolingbroke. Voltaire y parle de « l'estimable Gordon ${ }^{44}$ » qu'il place au même rang qu'un John Locke. L'anticléricalisme du penseur écossais n'a pu que trouver un écho sympathique chez celui qui voulait écraser «l'Infâme ».

14 La critique lancée par Gordon à l'égard de la religion est d'ailleurs relayée au même moment dans le journal de l'Abbé Prévost, Le Pour et le Contre, principalement consacré à la diffusion de la littérature et de la culture anglaises. Prévost s'intéresse à la brochure publiée par Gordon, A sermon preached at Lincoln'Inn ${ }^{45}$. Selon lui « on y voit régner la liberté de penser, qui fait son caractère, \& le clergé sur tout y est fort maltraitét ${ }^{46}$.

Ainsi, durant la première moitié du XVIII ${ }^{e}$ siècle, le traducteur de Tacite est connu en France, et ses idées sont discutées. Peut-on pour autant parler d'une influence de la philosophie politique de Gordon sur les penseurs français? Concernant Montesquieu par exemple, la question est loin d'être tranchée. Selon certains, l'auteur de l'Esprit des lois, qui connaissait les Cato's Letters, se serait inspiré des Discours sur Tacite au sujet du régime despotique, et des libertés individuelles ${ }^{47}$. Sur ces thèmes, les emprunts faits par Montesquieu à Gordon " pour être disséminés sans beaucoup d'ordre dans l'Esprit des Lois, n'en sont pas moins à la fois très nombreux et importants ${ }^{48}$. » Ailleurs, il est noté les affinités curieuses entre les considérations de Gordon sur les révolutions de Rome et 
celles de Montesquieu sur les révolutions de Chine ${ }^{49}$. De même, la méfiance nourrie par Gordon envers les armées professionnelles se retrouverait dans l'Esprit des Lois. ${ }^{50} \mathrm{La}$ corruption des monarchies selon Gordon commence par les Grands, et selon Montesquieu « le principe de la Monarchie se corrompt lorsque les premières dignités sont les marques de la première servitude ; lorsque l'on ôte aux grands le respect des peuples, et qu'on les rend de vils instruments du pouvoir arbitraire ${ }^{51}$.» A l'inverse, d'autres auteurs, comme Muriel Dodds dans l'entre-deux guerres ${ }^{52}$ ou plus récemment Charles Porset ${ }^{53}$ et Catherine Volpilhac-Auger, ont réfuté le fait que l'auteur Ecossais ait à ce point influencé Montesquieu, notamment dans l'interprétation que ce dernier fait de l'œuvre de Tacite. Selon Madame Volpilhac-Auger, « en France, après Montesquieu et d'Alembert, on n'a pas besoin de Gordon car l'essentiel est dit par des auteurs autrement plus écoutés que lui ${ }^{54}$. » Parmi les Lumières françaises, d'autres auteurs de premier plan se sont intéressés à l'œuvre gordonienne. Il en est ainsi d'Helvétius qui cite le penseur Ecossais dans son ouvrage De l'Esprit en $1759^{55}$. D'Alembert le mentionne plusieurs fois dans ses Morceaux choisis de Tacite en 1763, mais c'est simplement au travail de traducteur de Gordon qu'il y fait référence à quatorze reprises ${ }^{56}$. D'Holbach, en 1767, livre même une traduction de l' Independant Whig, prévenant que cet ouvrage est « du célèbre Thomas Gordon, connu pour son commentaire sur Tacite et sur Salluste, \& par beaucoup d'autres ouvrages favorables à la liberté civile et religieuse. Il fut aidé dans ses travaux généreux par M. Trenchard, membre du Parlement d'Angleterre \& zélé défenseur des droits de son pays ${ }^{57}$. » D'Holbach ne pouvait être que séduit par une œuvre qui critiquait l'organisation hiérarchique des religions anglicane et catholique ${ }^{58}$ et le pouvoir pontifical ${ }^{59}$. Bien entendu, l'Encyclopédie ne pouvait ignorer l'auteur écossais. On y trouve une référence à Gordon dans l'article "Terni », rédigé par le chevalier de Jaucourt, selon lequel les commentaires sur Tacite écrits par l'auteur britannique sont pleins de «fortes réflexions sur la liberté du gouvernement ${ }^{60}$.

17 Quelques voix se font discordantes cependant dans cette réception du penseur britannique. Ainsi, en 1764, dans sa Science du gouvernement, l'absolutiste Gaspard de Réal de Curban livre une notice assez complète dans laquelle il passe en revue l'essentiel des œuvres de Gordon. Selon de Réal, les observations politiques et morales de l'auteur ont le défaut d'être « fort communes et fort diffuses. ${ }^{61}$ » Il ajoute même, « lorsque l'auteur entre dans quelque détail, même sur les gouvernements modernes, il devient un forcené ; c'est un homme qui tombe en délire, qui calomnie plusieurs nations, qui déchire la réputation des plus grands princes \& qui adopte, sans aucune sorte de jugement, ce que les ennemis d'une nation ou des monarques ont dit dans ces libelles que le temps de la guerre fait éclore ${ }^{62}$.» On sent poindre derrière cette exécution en règle une certaine anglophobie, peut-être liée à l'humiliation du traité de Paris signée l'année précédente ! Au passage, la notice de Réal de Curban sera intégralement reprise par de Felice dans son Code de l'humanité en 1778 !

Dans les dernières années de l'Ancien Régime, l'œuvre de Gordon semble connaître un regain d'intérêt. Sans nul doute, l'invocation de Rome, qui revient en force dans les années 1780 , et les débats que suscite la guerre d'Amérique, peuvent expliquer cet engouement ${ }^{63}$. Quoi qu'il en soit, Gordon est souvent cité. Ainsi, Pierre Jean Grosley, dans son ouvrage intitulé Londres, écrit : « De tous les écrivains anglais qui m’ont passé sous les yeux, il n'en est aucun qui ait porté aussi loin que Gordon, l'austère rigidité sur les vertus qu'attend la patrie, \& qu'elle exige d'un véritable Anglais, surtout dans l'ordre de la noblesse ${ }^{64}$. » L'ouvrage référence des milieux parlementaires français, les Maximes du droit 
public, de Maultrot et Mey, publié en 1775, mentionne les Discours sur Tacite ${ }^{65}$. L'Encyclopédie méthodique de Panckoucke quant à elle rappelle que le livre de Gordon est " plein de fortes réflexions sur la liberté du gouvernement ${ }^{66}$. " Le Dictionnaire universel des sciences morales de Robinet dont la parution débute en 1777 et court jusqu'en 1783 fait une utilisation forcenée des écrits de l'auteur Ecossais. En effet, plusieurs articles y font référence: Abus, Affranchi, Arbitraire, Caton, Changements politiques, Citoyen, Courtisan, Domination, Gouverneur de Province, Indolence des princes, Liberté politique, Néron, Parti, Patricien, Plébéien, ou encore l'article Tribun ${ }^{67}$. Quant à Mirabeau, il utilise la méthode de Gordon dans son ouvrage sur la vie d'Agricola. Comme il le confie à Mme du Saillant en 1780, «J'aime Tacite à la passion, je l'ai tout traduit ici. J'y ai fourré des notes et des discours à la manière de Gordon ${ }^{68}$. » Dans son Essai sur le despotisme, Mirabeau avait d'ailleurs cité le traducteur de Tacite, notamment pour établir une identité entre despotisme et tyrannie antique ${ }^{69}$. Chez Mirabeau, la référence à Gordon n'est pas étonnante. En effet, le futur constituant semble avoir cédé aux sirènes du républicanisme classique et cette tradition nourrit son opposition à l'absolutisme ${ }^{70}$.

L'évocation de Mirabeau permettra de conclure par quelques mots sur la période révolutionnaire. Paru dans les premières années de la Révolution française, l'ouvrage de Maultrot, Origine et étendue de la puissance royale ${ }^{71}$, fait une place considérable aux principes politiques de Gordon rappelant que ce dernier « donne les idées les plus justes de la fin du gouvernement, qui est la borne nécessaire du pouvoir des princes ${ }^{72}$.» Selon Maultrot, « jamais auteur n'a été plus pénétré de la maxime, que le Roi est établi pour le bien du peuple, et non pour le sien propre $^{73}$.» Autre admirateur de Tacite, Camille Desmoulins utilise abondamment les Discours de l'auteur écossais désormais familier du public français. En 1794, dans le Vieux Cordelier, Desmoulins, alors aux côtés de Danton, se sert des commentaires de Gordon pour condamner la Terreur et prôner une République plus athénienne que celle rêvée par Robespierre et Saint-Just. En effet, « loin de penser que la liberté soit une égalité de disette, je crois au contraire qu'il n'est rien de tel que le gouvernement républicain pour amener la richesse des Nations [...] le commerce ne fleurit jamais dans un gouvernement despotique, parce que personne n'est assuré de jouir longtemps de ce qu'il possède, tandis que la liberté ne peut manquer d'éveiller l'industrie, et de porter les nations au plus haut degré ${ }^{74}$. " Dans les Discours sur Tacite, Gordon avait établi le lien entre le développement du commerce et la liberté en prenant l'exemple de Florence, " une république (...) sujette à des troubles fréquents, à des factions et à des partis. Cependant par le bonheur et la force de la liberté, le peuple y était nombreux, le commerce et l'art militaire y florissaient ${ }^{75}$.» Et lorsque Florence tomba entre les mains d'un prince, elle perdit son opulence en même temps que sa liberté ${ }^{76}$. Toutefois, en 1794, l'heure n'est pas encore à une République de «cocagne » comme le laisse entendre la terrible sentence de Saint-Just selon laquelle «il n'y a point de prospérité et de tranquillité à espérer tant que le dernier ennemi de la liberté respirera ${ }^{77}$.» Par ailleurs, Desmoulins, qui prône alors l'indulgence contre la Terreur, pense « avec Gordon, qu'il n'y eut jamais de secte, de société, d'église, de club, de loge, d'assemblée quelconque, de parti en un mot, tout composé de gens d'une exacte probité, ou entièrement mauvais, je crois

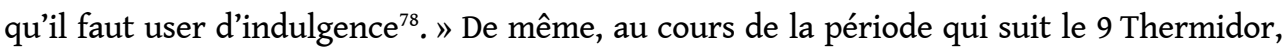
Gordon est cité dans le Mercure Français ${ }^{79}$. On y loue son exactitude en tant qu'historien de l'antiquité, et l'effort qu'il a nourri dans ses œuvres pour défendre la liberté publique ${ }^{80}$. Son œuvre est utilisée pour stigmatiser l'esprit de faction que les thermidoriens tentent alors d'associer au souvenir de Robespierre. Ainsi, « tous ceux qui suivent un chef de parti sont en général des gens perdus pour le corps de l'Etat. " Mais la République, même 
thermidorienne, reste en guerre contre la Grande-Bretagne, et malgré les éloges qui lui sont décernés, l'œuvre de Gordon "est d'un Anglais, partisan enthousiaste de la constitution de son pays, dont nous connaissons les vices nombreux [...] on doit prendre dans son livre (sur Tacite) que ce qui peut fortifier notre amour pour le républicanisme et pour les intérêts d'un peuple généreux, de citoyens, d'amis et de frères qui ont pris pour devise la liberté, l'unité, l'égalité, l'indivisibilité de la République ou la Mort ${ }^{81}$. C Comment affirmer plus clairement que si les Anglais ont pensé la République, les Français, eux, l'ont inscrite dans leurs institutions !

\section{NOTES}

1. Sur la question de la commonwealthman tradition, voir Rachel HAMMERSLEY, The English republican tradition and eighteenth-century France - Between the ancients and the moderns, Manchester et NewYork, Manchester University Press, 2010, p. 14-32.

2. Rachel HAMmERSLEY, "The Commonwealth of Oceana de James Harrington: un modèle pour la France révolutionnaire ", AHRF, 2005, n³42, p. 15 ; Blandine BARRET-KRIEGEL range Gordon dans un courant whig aux côtés de Bolingbroke (ce qui est surprenant puisque ce dernier est un tory) et Mandeville, «nourri aux idées du club de la Rota fondé par Harrington » ("L'influence politique et juridique de l'idée républicaine anglaise en France : Montesquieu et Rousseau » dans L'influence politique et juridique de l'Angleterre en Europe, Aix, PUAM, 2012, p. 69).

3. Comme l'a écrit Alexandra HYARD, «le républicanisme anglais du XVIII ${ }^{\mathrm{e}}$ siècle demande pour être compris un détour par le XVII ${ }^{\mathrm{e}}$ ( ( Adam Smith et le républicanisme », dans La République et l'idée républicaine en Grande-Bretagne, E-rea, 1.2, 2003, p. 2).

4. Alexis KELLER, «République ", dans Dictionnaire Montesquieu [en ligne], sous la direction de Catherine Volpilhac-Auger, ENS de Lyon, septembre 2013. URL: http://dictionnairemontesquieu.ens-lyon.fr/fr/article/1377636396/fr

5. Heather E. BARRY, A Dress Rehearsal of Revolution:John Trenchard and Thomas Gordon's works in eighteenth-century British America, University Press of America, 2007, p. 14.

6. Sur Gordon et Trenchard, voir Marie P. MCMAHON, The radical Whigs, John Trenchard and Thomas Gordon: libertarian Loyalists to the new House of Hanover, Lanham, Univerdsity Press of America, 1990.

7. Sous le titre l'Esprit du clergé ou le christianisme primitif vengé des entreprises et des excès de nos prêtres modernes, Londres, Marc Michel Rey, 1767.

8. Les Cato's Letters, or Essays on Liberty, Civil and Religious, and Other important Subjects, paraissent entre 1720 et 1723 dans le London Journal. Les 144 lettres sont ensuite réunies une première fois sous forme d'ouvrage à partir de 1723 à Londres chez Wilkins, Woodward, Walthoe and Peele.

9. Rachel HAMMERSLEY, The english republican tradition...., op.cit., p. 17.

10. Heather E. BARRY, A Dress Rehearsal of Revolution: John Trenchard and Thomas Gordon's works in eighteenth-century British America, op.cit., p. 16.

11. Rachel HAMMERSLEY, The english republican tradition..., op.cit., p. 59.

12. Vincenzo FERRONE, La politique des Lumières: Constitutionnalisme, républicanisme, droits de l'homme, le cas Filangieri, Paris, L'Harmattan, 2010, p. 166. L'auteur explique que dans les Cato's Letters, la tradition républicaine est rapprochée de la pensée jusnaturaliste de Locke. Le discours politique de Gordon était destiné à «nourrir la lutte politique en Angleterre » mais également 
plus tard les révolutionnaires américains «dans leur recherche d'un républicanisme adapté à l'époque moderne " (Ibid).

13. Thomas GORDON, The Works of Tacitus With Political Discourses, volume I, London, Woodward and Peele, 1728. L'ouvrage s'ouvre par un hommage appuyé à Walpole dans une dédicace de trois pages.

14. Thomas GORDON, The Works of Sallust, London, Woodward and Peele, 1744.

15. Eugène et Emile HAAG, La France protestante, Paris et Genève, Cherbuliez, t. IV, 1858, p. 207 sq. Pierre Daudé est né en 1681 à Marvejols et mort en 1754 vraisemblablement à Londres. Il fait partie du Refuge Huguenot. Il est collaborateur de la Bibliothèque Britannique et entretient une correspondance avec Des Maizeaux.

16. Thomas GORDON, Discours historiques, critiques et politiques sur Tacite, traduit de l'anglais par Pierre Daudé, Amsterdam, François Changuion, 1742, p. 3.

17. Arrest de la souveraine cour de Parlement du vingt-sixiéme août mil sept cens cinquante-un qui ordonne que la livre intitulé Discours historiques, critiques \& politiques sur Tacite sera laceré \& brûlé par la main de l'executeur de la Haute Justice, Toulouse, édition Veuve de Claude-Gilles Lecamus, 1752, p. 45.

18. Sur l'engouement du XVIII ${ }^{\mathrm{e}}$ siècle français pour l'histoire romaine, voir Claude NICOLET, La fabrique d'une nation. La France entre Rome et les Germains, Paris, Perrin, 2003, p. 15-39.

19. Ibid, p. 22.

20. Mouza RASKOLNIKOFF, Histoire romaine et critique historique dans l'Europe des Lumières, Strasbourg, AECR, 1992, p. 343-344.

21. Bibliothèque britannique ou Histoire des ouvrages des savans de la Grande-Bretagne, avril-juin 1733, Pierre de Hondt, La Haye, p. 36-60.

22. Ibid., p. 36.

23. Ibid., p. 37.

24. Ibid., p. 38.

25. Ibid., p. 45 : «L'Auteur met dans tout son jour le droit qu'ont les peuples de résister aux tyrans. [...] La conduite des mauvais empereurs romains, leurs barbaries, \& leurs fureurs continuelles, sont capables d'autoriser les principes les plus antimonarchiques. »

26. Ibid., p. 52: «Les troupes ont beau être disciplinées; plus la discipline d'une armée est excellente plus elle est à craindre pour la liberté de l'Etat. L'union des officiers \& des soldats les met en état d'exécuter tous les projets de leurs chef \& il est rare qu'il n'en forme pas de contraires aux intérêts de la patrie ».

27. Louis DE JAUCOURT, Armand BOISBELEAU DE LA CHAPELLE, Bibliothèque raisonnée des ouvrages des savans de l'Europe, volume 28, Wetstein éditeur, Amsterdam, 1742.

28. Edouard TILLET, La constitution anglaise, un modèle politique et institutionnel dans la France des Lumières, Aix, PUAM, 2001, p. 51-64.

29. Pierre Daudé a publié en 1742 une première traduction des Works of Tacitus à Amsterdam chez l'éditeur François Changuion. Celle des Works of Sallust paraît en 1759. Elle est souvent donnée sans indication de lieu. Heather E. HEARY pense qu'elle a été éditée chez Cramer à Genève (A Dress Rehearsal of Revolution: John Trenchard and Thomas Gordon's works..., op.cit., p. 26-27).

30. Louis DE JAUCOURT, Armand BOISBELEAU DE LA CHAPELLE, Bibliothèque raisonnée des ouvrages des savans de l'Europe..., op.cit., p. 375.

31. Ibid., p. 382.

32. Ibid. : « Voilà Mr. Gordon entièrement dans les idées de Mr. Bayle. »

33. Ibid., p. 383.

34. Ibid., p. 389.

35. Ibid.

36. Ibid. 
37. Ibid., p. 389-390.

38. Ibid., p. 391.

39. Thomas GORDON, Discours historiques, critiques et politiques sur Tacite, traduit de l'anglais par Pierre Daudé,..., édition de 1742, op.cit., p. 195.

40. Louis DE JAUCOURT, Armand BOISBELEAU DE LA CHAPELLE, Bibliothèque raisonnée des ouvrages des savans de l'Europe..., op.cit., p. 395.

41. voltaire, Lettres philosophiques, Amsterdam, E. Luca éditeur, 1734, p.113. En effet, RapinThoiras est l'auteur d'une volumineuse Histoire d'Angleterre parue à La Haye entre 1724 et 1727, et dans laquelle il ne cite pas Gordon.

42. Correspondance de Voltaire avec le roi de Prusse, Librairie de la Bibliothèque Nationale, Paris, 1889, p. 81: «Votre Altesse Royale me dit qu'il lui a fallu lire bien des livres pour son AntiMachiavel, tant mieux, car elle ne lit qu'avec fruit ; ce sont des métaux qui deviendront or dans votre creuset. »

43. voltaire, Histoire de l'établissement du christianisme, dans Euvres Complètes, Paris, Renouard, tome 2, 1819, p. 243.

44. Ibid., p. 360.

45. Paru sous forme anonyme à Londres chez l'éditeur Peele en 1732.

46. Abbé Antoine-François PREVOST, Le Pour et le Contre: ouvrage périodique d'un goût nouveau, La Haye, Isaac van der Kloot, t. 2, 1734, p. 262.

47. C'est l'opinion de Joseph DEDIEU qui consacre une thèse à l'influence anglaise sur la pensée de Montesquieu en 1909.

48. Joseph DEDIEU, Montesquieu et la tradition politique anglaise en France: les sources anglaises de l'Esprit des Lois, Genève, Slatkine reprint, 1970, p. 284.

49. Ibid., p. 297.

50. Ibid., p. 300.

51. Ibid., p. 303.

52. Dans son livre paru en 1929, Muriel DoDDs s'efforce de minimiser l'influence qu'aurait pu exercer Gordon sur Montesquieu (Les récits de voyage: sources de l'Esprit des Lois de Montesquieu, Genève, Slatkine, 1929, p. 138-149).

53. Charles PORSET, «Despotisme: du mot à l'histoire », dans Simone GOYARD-FABRE (dir.), L'Etat moderne: 1715-1848, Paris, Vrin, 2000, p. 57.

54. Catherine volPilHaC-AUger, Tacite en France de Montesquieu à Chateaubriand, Oxford, Voltaire Foundation of the Taylor institution, p. 433.

55. Helvetius, De l'Esprit, Paris, Chez Durand, 1759, p. 396.

56. Jean LE ROND D'ALEMBERT, Morceaux choisis de Tacite traduits en Français avec le latin à côté, Lyon, Bruyset éditeur, 1763.

57. Paul Henry Thiry D’HоLBACH (traducteur), L'esprit du clergé ou le christianisme primitif vengé des entreprises et des excès de nos prêtres modernes, op.cit., p. 2.

58. Thomas GORDON, John TRENCHARD, Independant whigh, or a defense of the primitive Christianity, London, printed for Peele, volume I, 1735, p. X-XI : « There is no Medium between Popery and Reformation; that is, between the claiming of any power in religion, and the the renouncing of all power in religion. "

59. Selon Gordon et Trenchard : «The Church of Rome was the Spiritual Babylon (...) and the Pope was Anti-Christ » (Ibid, p. 143).

60. ARTFL, Encyclopédie Project, p. $16: 161$.

61. Gaspard REAL DE CURBAN, La science du gouvernement, Amsterdam, Arkstée et Merkus éditeurs, 1764 , tome VIII, p. 661.

62. Ibid.

63. Claude NICOLET, La fabrique d'une nation..., op.cit., p. 37. 
64. Pierre Jean GROSLEY, Londres, Lausanne, t. 2, 1774, p. 45.

65. Gabriel Nicolas MAULTROT, Claude MEY, Maximes du droit public Français, tirées des capitulaires, des ordonnances du Royaume et autres monuments de l'Histoire de France, Amsterdam, Marc-Michel Rey, 1775 , t. 1 , p. 374 et p. 390.

66. Charles-Joseph Panckoucke, Encyclopédie Méthodique, Paris, Panckoucke, t. 3, 1788, p. 365.

67. Jean-Baptiste ROBINET, Dictionnaire universel des sciences morale, économique, politique et diplomatique ou Bibliothèque de l'homme-d'Etat et du citoyen, Londres, 1783.

68. Lettre de Mirabeau à Madame du Saillant du 11 septembre 1780, citée par François QUASTANA, La pensée politique de Mirabeau (1771-1789) : Républicanisme classique et régénération de la monarchie, Aix, PUAM, 2007, p. 70.

69. Euvres choisies de Mirabeau, Paris, Brissot-Thivars, 1821, p. 69 : «Un despote, dit Gordon, ne fait que renouveler les prétentions surannées des anciens tyrans. » Pour François QUASTANA, les différents emprunts faits par Mirabeau aux traductions gordonniennes de Tacite et Salluste « constituent une preuve tangible de l'importance de la contribution idéologique trop souvent méconnue fournie aux Lumières françaises tardives par une sociologie de la liberté et par un discours civique développés à partir des moralistes romains, revivifiés par Machiavel, puis transmis à la pensée britannique sous la forme de l'idéologie du pays et disponible pour la pensée française anglomane » (La pensée politique de Mirabeau..., op.cit., p. 71).

70. Ibid., p. 16.

71. Gabriel-Nicolas MAULTROT, Origine et étendue de la puissance royale selon les livres saints et la tradition, vol. 3, Paris, Le Clère, 1789-1790.

72. Ibid., p. 317.

73. Ibid., p. 405.

74. Camille Desmoulins, Le vieux cordelier, Paris, Ebrard, 1834, p. 144-145. Il est également intéressant de comparer les propos de Desmoulins à ceux de Gordon et Trenchard: «Les privilèges de penser, de dire, de faire ce qui nous plait, et de devenir aussi riche que l'on peut, avec pour seule restriction qu'en faisant tout cela nous ne heurtons ni le public, ni les uns les autres, sont les glorieux privilèges de la liberté; et ses effets sont la vie en liberté, dans l'abondance et la sécurité (...) tout bonheur et toute prospérité civiques sont inséparables de la liberté » (Cato's Letters, Indianapolis, Liberty Fund, 1990, t. I, p. 432).

75. Thomas GORDON, Discours historiques, critiques et politiques..., édition de 1742, op.cit., p. 195-196.

76. Ibid., p. 196.

77. Rapport fait devant la Convention au nom du Comité de Salut Public le 19 vendémiaire an II.

78. Camille DESMOULINS, Le vieux cordelier..., op.cit., p. 181.

79. Mercure Français, Historique, Politique et Littéraire; par une société de patriotes, Paris, t. XII, 16 septembre 1794 , p. 97 sq.

80. Ibid.

81. Ibid.

\section{RÉSUMÉS}

L'œuvre de Gordon, notamment ses traductions de Tacite et de Salluste, est reçue en France au cours du XVIII ${ }^{e}$ siècle. Elle est abondamment commentée par les lettrés français qui s'intéressent alors à l'histoire romaine. Elle nourrit de même les opposants à l'absolutisme qui relaient les 
principes républicains de l'auteur britannique. A l'inverse, ceux-ci sont dénoncés par les tenants de la monarchie absolue à l'instar de Réal de Curban. Gordon inspire également les révolutionnaires français, comme Mirabeau et Desmoulins.

Gordon's writings, in particular his translations of Tacitus and Sallust, reached France during the eighteenth century. They were commented on at length by French men of letters who took an interest in Roman history. These writings also fuelled opponents of absolutism who relayed the British author's republican principles. Conversely, these writings were condemned by disciples of absolute monarchy such as Réal de Curban. Gordon also inspired French revolutionaries such as Mirabeau and Desmoulins.

INDEX

Keywords : Thomas Gordon, reception, France, eighteenth century, French Revolution

Mots-clés : Thomas Gordon, réception, France, XVIIIe siècle, Révolution française

\section{AUTEUR}

ERIC GASPARINI

CERHIIP - EA 2186

Eric.gasparini@free.fr 